\title{
A Novel Bidirectional DC-DC Converter with High Efficiency and Small Size AC Link
}

\author{
Katsuji Iida $^{1}$, Hirofumi Matsuo ${ }^{2}$, Toshiro Hirose ${ }^{2}$, Yoichi Ishizuka ${ }^{2}$ \\ ${ }^{1}$ Komatsu Ltd, Kanagawa, 254-8567, Japan \\ ${ }^{2}$ Nagasaki University, Nagasaki, 852-8521, Japan \\ E-mail: h-matsuo@nagasaki-u.ac.jp
}

\begin{abstract}
Recently, it is required that the high efficiency and small size DC-AC inverter and DC-DC converter are developed for the applications such as solar cells, fuel cells and secondary batteries in the telecommunications, home electronics, industries, electric cars and so forth. A novel bidirectional DC-DC converter is proposed and developed, which realizes the small size and high efficiency $98 \%$ is achieved in the prototype bidirectional DC-DC converter.
\end{abstract}

\section{INTRODUCTION AND BACKGROUND}

Advancement in semiconductor device technology has been driving the rapid diffusion of high voltage, high efficiency compact power converter systems (incl. inverters), in contrast with fuel batteries and secondary batteries which have made little headway toward practical high voltage systems due to poor cost performance. This trend makes it possible to proactively use DC-DC converters which transmit DC power bidirectionally (i.e. during both power regenerating and running modes) ${ }^{[1]}$, in order for an electric energy shifting to take place between different supply voltages.

However, there is no point if a high voltage inverter aiming at improved efficiency is counteracted by the power loss and increased weight of a DC-DC converter, thus, requiring actualization of high efficiency and compact DC-DC converters.

A typical DC-DC converter, or second-quadrant chopper (i.e. reversible chopper) ${ }^{[1]}{ }^{[2]}$, is shown in Fig.1 and a bidirectional isolated DC-DC converter ${ }^{[3]}$ is also in common

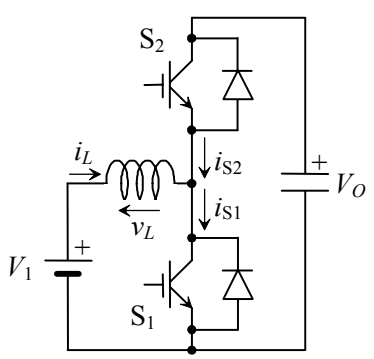

Fig. 1 2-quadrant chopper use, as shown in Fig. 2. In these kinds of converters, total power transferred goes through the circuits. The switching element voltage of the circuits is controlled on the high-voltage side, while current is controlled on the low-voltage side. This causes a high-voltage and large-current condition, allowing for an increase in both conduction loss and switching loss. It results in a limitation in efficiency improvement.

Devices such as transformers and inductors require costly manpower for their installation, compared with low-price semiconductor devices. This situation urges us to establish a cost-effective converter circuit mode.

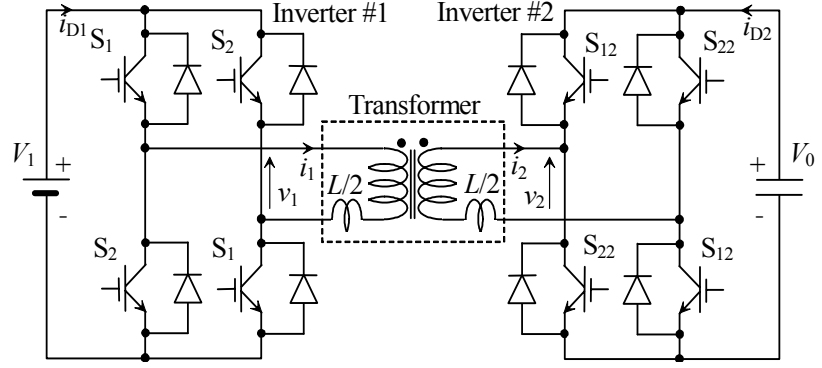

Fig. 2 Isolated bidirectional dc-dc converter

To solve these problems, an AC-link bidirectional DC-DC converter is proposed, which is composed of two voltage source inverters and an AC transformer, as shown in Fig. 3. The transformer is not used to isolate the input and output power circuit. DC terminals of inverters \#1 and \#2 are connected to the additive polarity in series, while their AC terminals are connected with the transformer with an appropriate leakage inductance. The phase difference between inverters $\# 1$ and $\# 2$, which are driven in one-pulse mode at 180 degree conduction, are controlled. This control strategy enables the determination of not only the direction of the power transmitted but also amount of the power transmitted. The maximum power can be achieved at a phase difference of 90 degrees during both power regenerating and running modes, which depends on the circuit parameters.

The main feature of the proposed circuit is that the power processed by two inverters and a transformer is only half of the electric power transmitted. The output voltage (Vo) shared with the inverters \#1 and \#2. It contributes to lower the voltage rating of the inverter switching elements.

In this new circuit in Fig. 3 as well as the conventional circuit illustrated in Fig. 2, the zero-current switching (ZCS) and zero-voltage switching (ZVS) are realized under the wide operation range when all switching elements are turned on. The high-efficiency operation of the proposed circuit is achieved by this realization of the ZCS and ZVS. The power to be processed by the transformer is decreased by half. Furthermore, only the AC current flows through the transformer in the proposed circuit in Fig.3. However, the DC current flows through the inductor in the conventional circuit in Fig.1. For this AC mode operation size and weight of the proposed DC-DC converter can be minimized. 


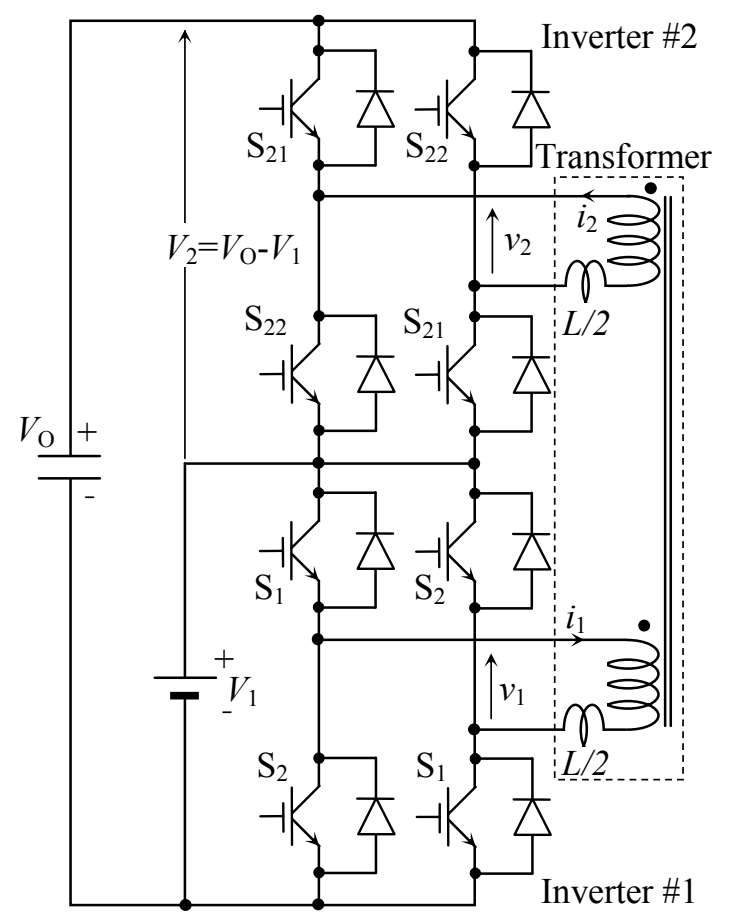

Fig. 3 Proposed ac link bidirectional dc-dc converter

\section{Operating Mode}

The circuits illustrated in Figs. 1 through 3 are boost-type DC-DC converters in which their power sources are on the low-voltage side. However, since DC-DC converters transmit

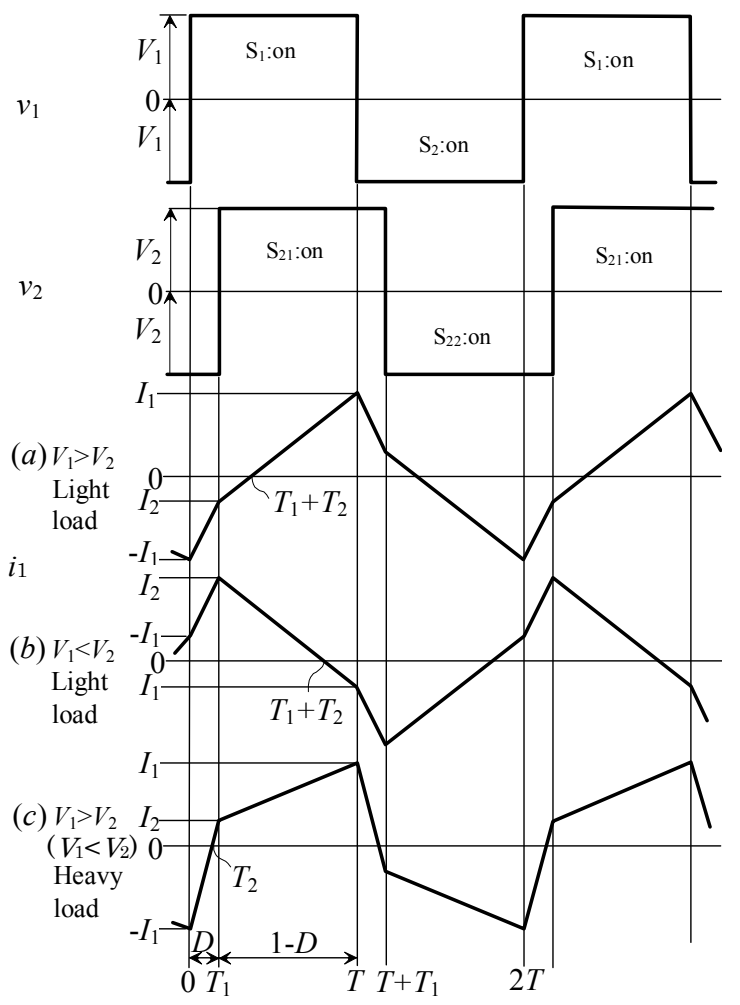

(a) Power running mode
DC power bidirectionally, their circuits operate as back-type DC-DC converters when the power source is replaced with load.

In this paper, it should be assumed that, in boost-type DCDC converters, the DC power source voltage of inverter \#1 is equal to that of inverter \#2, the turn ratio of the primary and secondary windings of the transformer is unity and Vo is equal to $2 \mathrm{Vi}$. Here, the operation of the power transmitted from V1 toward $\mathrm{Vo}$ is in power running mode, while its reverse operation is in power regenerative mode. An individual inverter is driven in one-pulse mode without controlling pulse width. The inverter is designed so that the phase of inverter \#1 on the low-voltage side advances against that of inverter \#2 on the high-voltage side in power running mode, while the phase of inverter \#1 lags behind that of inverter \#2 in power regenerative mode. The primary current $i 1(=$ secondary current $i 2$ ) of a transformer has three patterns of current waveforms, depending on the currents $(i 1, i 2)$ of DC power sources as well as the voltage $(V 1, V 2)$ of DC power source. During both power regenerative and running modes, as shown in Fig. 4 (c), the state of ZCS and ZVS is created because an anti-parallel diode is in a conduction state, resulting in the turn-on of all switching elements after the dead-time. However, S21 and S22 in the power running mode (a), S1 and S2 in the power running mode (b), S1 and S2 in the power regenerative mode (a) and S21 and S22 in the power regenerative mode (b) are turn-on in the hard-switching operation. In this hardswitching operation, since the load current is relatively low, the switching power loss is small during the recovery time internal of the diode.

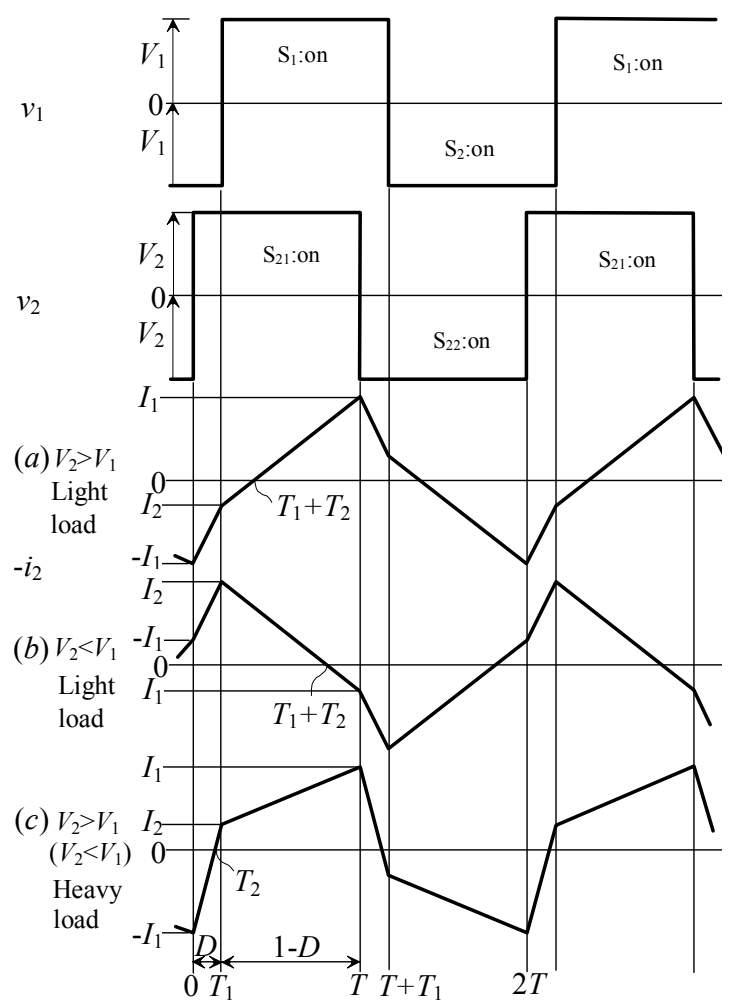

(b) Power regenerative mode 


\section{OPERATING ANALYSIS}

Operating analysis of the proposed DC-DC converter is performed, based on numerical analysis taking into account the conduction loss of switching elements, the resistance of

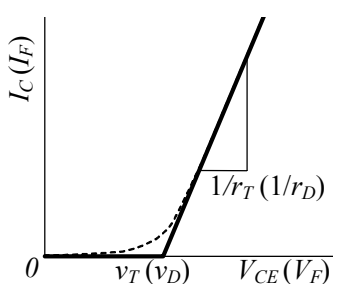

Fig. 5 Model of device the transformer, including DC resistance, skin effect, $\mathrm{AC}$ resistance such as eddy current loss, and the internal resistance of the DC power source. Then, the switching loss and the transformer iron loss are added to the power on the power transmitting side.

Fig. 5 shows the approximated model of semiconductor devices, that is, IGBT and diode. Table 1 shows the symbols used in the circuit, and circuit parameters.

Furthermore, the followings assumed in the analysis:

1) The resistance of the semiconductor devices are infinite when they are in off-state.

2) The transformer excitation current is ignored because of its gapless construction and large mutual inductance.

Table 1 Circuit symbols and parameters

\begin{tabular}{|l|c|c|}
\hline IGBT threshold voltage & $\mathrm{v}_{\mathrm{T}}$ & $0.8 \mathrm{~V}$ \\
\hline Diode threshold voltage & $\mathrm{v}_{\mathrm{D}}$ & $0.9 \mathrm{~V}$ \\
\hline IGBT on-resistance & $\mathrm{r}_{\mathrm{T}}$ & $2.05 \mathrm{~m} \Omega$ \\
\hline Diode on-resistance & $\mathrm{r}_{\mathrm{D}}$ & $3.5 \mathrm{~m} \Omega$ \\
\hline $\begin{array}{l}\text { Total resistance of } \\
\text { transformer }\end{array}$ & $\mathrm{r}_{\mathrm{L}}$ & $80 \mathrm{~m} \Omega$ \\
\hline $\begin{array}{l}\text { Internal resistance of } \\
\text { low voltage source }\end{array}$ & $\mathrm{r}_{1}$ & $1 \mathrm{~m} \Omega$ \\
\hline $\begin{array}{l}\text { Internal resistance of } \\
\text { high voltage source }\end{array}$ & $\mathrm{r}_{0}$ & $1 \mathrm{~m} \Omega$ \\
\hline $\begin{array}{l}\text { Leakage inductance of } \\
\text { transformer }\end{array}$ & $\mathrm{L}_{2}$ & $23 \mu \mathrm{H}$ \\
\hline Voltage of transformer & $\mathrm{v}_{\mathrm{M}}$ & $275 \mathrm{~V}=\mathrm{V}_{0} / 2$ \\
\hline Switching frequency & $\mathrm{f}$ & $10 \mathrm{kHz}$ \\
\hline $\begin{array}{l}\text { Direct voltage source } \\
\text { in low voltage side }\end{array}$ & $\mathrm{V}_{1}$ & $275 \mathrm{~V}$ \\
\hline $\begin{array}{l}\text { Direct voltage source } \\
\text { in high voltage side }\end{array}$ & $\mathrm{V}_{0}$ & $550 \mathrm{~V}$ \\
\hline
\end{tabular}

Thus, because the turn ratio of the transformer is unity, primary current $i 1$ is equal to secondary current $i 2$. In this paper detailed analysis is given for the power running mode (a), while only the results are given for other modes.

\subsection{State $a$ (time $t=0$ to $T 1$ )}

In power running mode (a): leakage inductor magnetic energy discharging period 1 :

After S2 arm (IGBT) and S22 arm (diode) are in on-state, $\mathrm{S} 2$ arm is turned off at time $t=0$, then $i 1 \mathrm{a}=i 2 \mathrm{a}=-I 1$ and state a is starting. Since $i 1 \mathrm{a}$ and $i 2 \mathrm{a}$ cannot be changed suddenly due to the leakage inductor magnetic energy, $i 1 \mathrm{a}$ flows to the S1 arm (diode) while i2a flows to the S22 arm (diode) continuously. Thus, when S1 arm (IGBT) is turned off after the dead time, current flows to the anti-parallel diode, leading to the states of ZVS and ZCS. No turn-on switching power loss is caused.

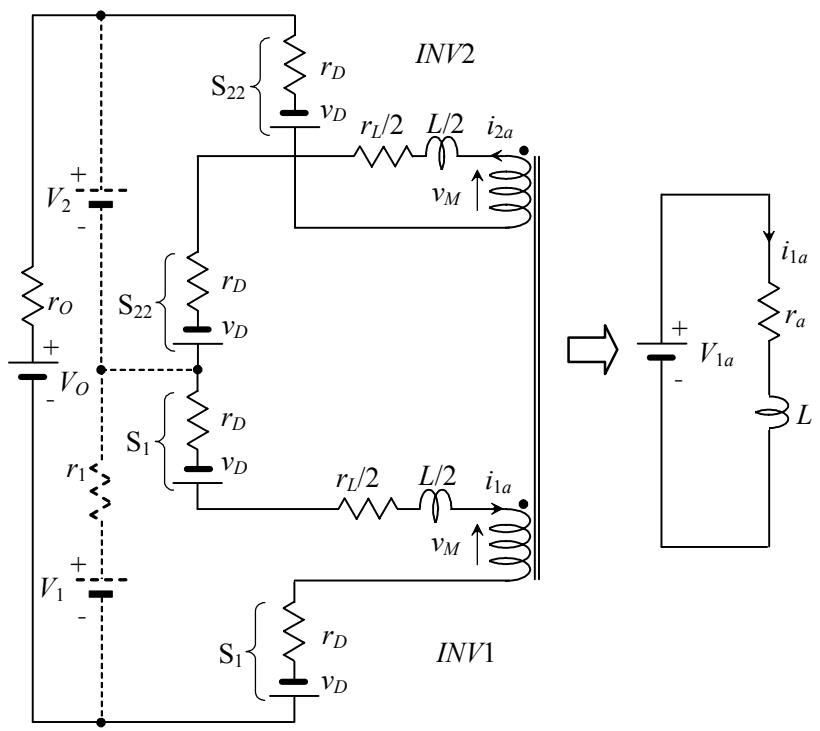

Fig. 6 Current-route and it's equivalent circuit in state a

Fig. 6 shows the current route of state $a$, and its equivalent circuit. In state $a$, current does not flow through $V 1$. Each initial value of $i 1 \mathrm{a}$ and $i 2 \mathrm{a}$ is defined as $-I 1$. Based on the current route, the following Laplace simultaneous equations are obtained.

$$
\begin{array}{r}
\frac{V_{1}}{s}=\left(\frac{L s+r_{L}}{2}+2 r_{D}\right) I_{1 a}(s)-\frac{2 v_{D}}{s}+\frac{L I_{1}}{2}+V_{M}(s) \\
\frac{V_{O}}{s}=\left(\frac{L s+r_{L}}{2}+2 r_{D}+r_{O}\right) I_{2 a}(s)-\frac{2 v_{D}}{s}+\frac{L I_{1}}{2}-V_{M}(s)+\frac{V_{1}}{s}
\end{array}
$$

Here, $i 1 \mathrm{a}=i 2 \mathrm{a}$. When $V M(s)$ is eliminated from the equations (3.1.1) and (3.1.2), the following equations are obtained.

$$
\begin{gathered}
I_{1 a}(s)=I_{2 a}(s)=\frac{V_{O}+4 v_{D}-L I_{1} s}{L s\left(s+\frac{r_{L}+r_{O}+4 r_{D}}{L}\right)}=\frac{\frac{V_{1 a}}{r_{a}}}{s}-\frac{\frac{V_{1 a}}{r_{a}}+I_{1}}{s+\frac{r_{a}}{L}} \\
\therefore i_{1 a}(t)=i_{2 a}(t)=\frac{V_{1 a}}{r_{a}}-\left(\frac{V_{1 a}}{r_{a}}+I_{1}\right) e^{-\frac{r_{a}}{L} t}
\end{gathered}
$$

Where,

$$
r_{a}=r_{L}+r_{O}+4 r_{D}, V_{1 a}=V_{O}+4 v_{D}
$$

The equivalent circuit of state $a$ is illustrated in Fig. 6. The current flow toward the diode at time $T 1$ is continued in this 
state. In the condition $(T 1 / T=D)$ as the current is defined as $I 2$ (negative), the following equation is obtained.

$$
e^{-\frac{r_{a}}{L} T_{1}}=e^{-\frac{\pi r_{a}}{\omega L} D}=\frac{\frac{V_{1 a}}{r_{a}}-I_{2}}{\frac{V_{1 a}}{r_{a}}+I_{1}}
$$

\subsection{State $b$ (Time $t=T 1$ to $T 1+T 2$ )}

In power running mode $(a)$ : leakage inductor magnetic energy discharging period 2 :

At time $T 1$, a turn-off signal is applied to S22 arm. Then, since current flows to the anti-parallel diode, no turn-off switching power loss is caused. And leading to the states of ZVS and ZCS. Therefore, S22 arm is continuously in conduction state.

S21 arm (IGBT) is turned on after the dead time, and a reverse recovery current flows for the reverse recovery time of diode of S22 arm, when there exists the short-circuit of DC power source $V 2$. This short circuit causes switching losses of both IGBT and diode. However, such losses are relatively minor because state $b$ appears under only the light load condition. The leakage inductor magnetic energy gradually decays, and then becomes zero at time $(T 1+T 2)$. Finally, state $b$ ends. Fig. 7 shows the current route of state $b$, and its equivalent circuit.

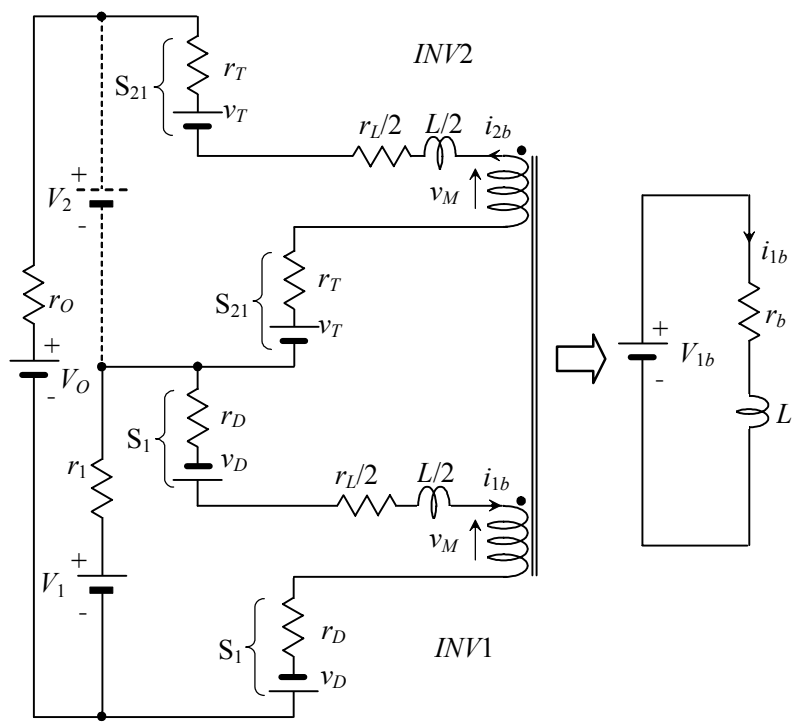

Fig. 7 Current-route and it's equivalent circuit in state b

In state $b$, currents $i 1 \mathrm{~b}$ and $i 2 \mathrm{~b}$ flow through $V 1$. Each initial value of $i 1 \mathrm{~b}$ and $i 2 \mathrm{~b}$ is defined as $I 2$ (negative). Under the condition that the starting time of state $b$ is defined as $t=0$ because of simple representation, the following Laplace simultaneous equations are obtained, based on the current route.

$$
\frac{V_{1}}{s}=r_{1}\left\{I_{1 b}(s)+I_{2 b}(s)\right\}+\left(\frac{L s+r_{L}}{2}+2 r_{D}\right) I_{1 b}(s)-\frac{2 v_{D}}{s}-\frac{L I_{2}}{2}+V_{M}(s)
$$

$$
\frac{V_{O}}{s}=-r_{1}\left\{I_{1 b}(s)+I_{2 b}(s)\right\}-\left(\frac{L s+r_{L}}{2}+2 r_{T}+r_{O}\right) I_{2 b}(s)+\frac{2 v_{T}}{s}+\frac{L I_{2}}{2}+V_{M}(s)+\frac{V_{1}}{s}
$$

Here, $i 1 \mathrm{~b}=i 2 \mathrm{~b}$. When $V M(s)$ is eliminated from the equations (3.2.1) and (3.2.2), the following equations are obtained:

$$
\begin{gathered}
I_{1 b}(s)=I_{2 b}(s)=\frac{2 V_{1}-V_{O}+2\left(v_{T}+v_{D}\right)+L I_{2} s}{L s\left(s+\frac{r_{L}+2\left(r_{T}+r_{D}\right)+r_{O}+4 r_{1}}{L}\right)}=\frac{\frac{V_{1 b}}{r_{b}}}{s}-\frac{\frac{V_{1 b}}{r_{b}}-I_{2}}{s+\frac{r_{b}}{L}} \\
\therefore i_{1 b}(t)=i_{2 b}(t)=\frac{V_{1 b}}{r_{b}}-\left(\frac{V_{1 b}}{r_{b}}-I_{2}\right) e^{-\frac{r_{b} t}{L} t}
\end{gathered}
$$

Where,

$$
\begin{aligned}
& r_{b}=r_{L}+2\left(r_{T}+r_{D}\right)+r_{O}+4 r_{1} \\
& V_{1 b}=2 V_{1}-V_{O}+2\left(V_{T}+V_{D}\right)
\end{aligned}
$$

The equivalent circuit of state $b$ is illustrated in Fig. 7. The current becomes zero at time $(T 1+T 2)$. State $b$ ends.

$$
e^{\frac{r_{b}}{L} T_{2}}=e^{\frac{\pi_{b} T_{2}}{\omega L} \frac{r^{2}}{T}}=1-\frac{r_{b} I_{2}}{V_{1 b}}
$$

\subsection{State $c$ (Time $t=T 1$ to $T 1+T 2$ )}

In power running mode $(a)$ : leakage inductor magnetic energy charging period:

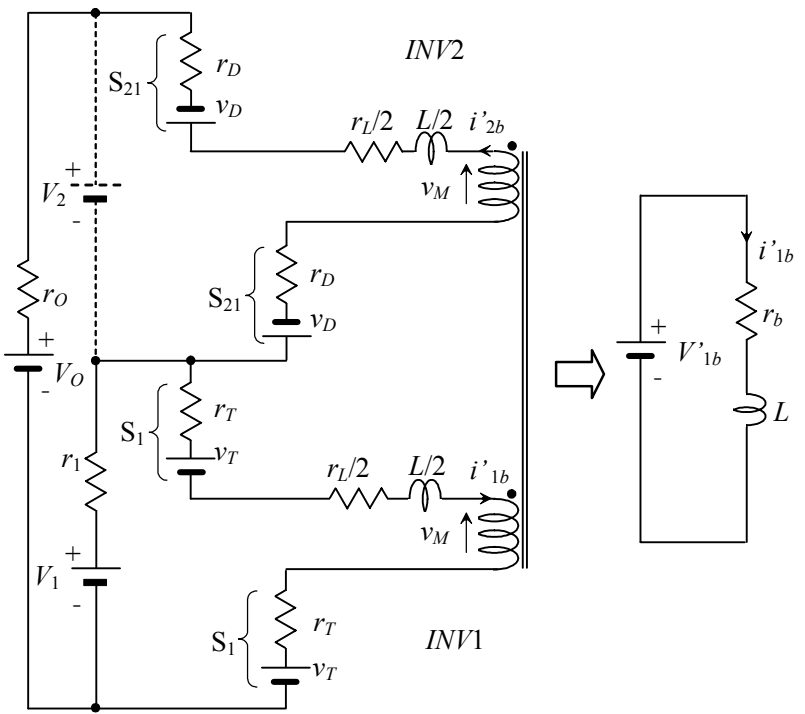

Fig. 8 Current-route and it's equivalent circuit in state c

After the magnetic energy of the leakage inductor diminishes at time $(T 1+T 2)$ and the current then becomes zero, $\mathrm{S} 1$ arm (IGBT) is turned on. Thus, current flows through S1 arm (IGBT) and the leakage inductor is excited by reverse polarity, resulting in current flow in the opposite direction. By contrast, current also flows through S21 arm (diode). In this case, since the reverse current naturally occurs, no switching loss is caused. 
Magnetic energy with reverse polarity is accumulated in the leakage inductor. At time $T, \mathrm{~S} 1$ arm (IGBT) is turned off. Then, state $c$ ends, and the positive half cycle concurrently ends. After that, the negative half cycle begin. Although the switching elements are exchanged, its operation mechanism is similar to that in the positive half cycle.

Fig. 8 shows the current route of state $c$, and its equivalent circuit. Each initial value of $i^{\prime} 1 \mathrm{~b}$ and $i^{\prime} 2 \mathrm{~b}$ are zero. Under the condition that the starting time of state $c$ is defined as $t=0$ because of simple representation. The following Laplace simultaneous equations are obtained, based on the current route.

$$
\begin{aligned}
& \frac{V_{1}}{s}=r_{1}\left\{I_{1 b}^{\prime}(s)+I_{2 b}^{\prime}(s)\right\}+\left(\frac{L s+r_{L}}{2}+2 r_{T}\right) I_{1 b}^{\prime}(s)+\frac{2 v_{T}}{s}+V_{M}(s) \\
& \frac{V_{O}}{s}=-r_{1}\left\{I_{1 b}^{\prime}(s)+I_{2 b}^{\prime}(s)\right\}-\left(\frac{L s+r_{L}}{2}+2 r_{D}+r_{O}\right) I_{2 b}^{\prime}(s)-\frac{2 v_{D}}{s}+V_{M}(s)+\frac{V_{1}}{s}
\end{aligned}
$$

Here, $i^{\prime} 1 \mathrm{~b}=i^{\prime} 2 \mathrm{~b}$. When $V M(s)$ is eliminated from equations (3.3.1) and (3.3.2), the following equations are obtained.

$$
\begin{gathered}
I_{1 b}^{\prime}(s)=I_{2 b}^{\prime}(s)=\frac{2 V_{1}-V_{O}-2\left(v_{T}+v_{D}\right)}{L s\left(s+\frac{r_{L}+2\left(r_{T}+r_{D}\right)+r_{O}+4 r_{1}}{L}\right)}=\frac{\frac{V_{1 b}^{\prime}}{r_{b}}}{s}-\frac{\frac{V_{1 b}^{\prime}}{r_{b}}}{s+\frac{r_{b}}{L}} \\
\therefore i_{1 b}^{\prime}(t)=i_{2 b}^{\prime}(t)=\frac{V_{1 b}^{\prime}}{r_{b}}\left(1-e^{-\frac{r_{b}}{L} t}\right)
\end{gathered}
$$

Where,

$$
\begin{aligned}
& r_{b}=r_{L}+2\left(r_{T}+r_{D}\right)+r_{O}+4 r_{1} \\
& V^{\prime}{ }_{1 b}=2 V_{1}-V_{O}+2\left(V_{T}+V_{D}\right)
\end{aligned}
$$

The equivalent circuit of state $c$ is illustrated in Fig. 8. Since $i$ ' $1 \mathrm{~b}$ in steady state becomes $I 1$ at time $T$, the following equation is obtained from equation (3.3.4).

$$
I_{1}=\frac{V_{1 b}^{\prime}}{r_{b}}\left\{1-e^{-\frac{r_{b}}{L}\left(T-T_{1}-T_{2}\right)}\right\}=\frac{V_{1 b}^{\prime}}{r_{b}}\left(1-e^{-\frac{\pi_{b}}{\omega L}(1-D)} e^{-\frac{\pi_{b} T_{2}}{\omega L} T}\right)
$$

\subsection{Operations in power running mode (a)}

\subsubsection{Determination of $I 1$ and $I 2$}

$I 1$ and $I 2$ are given by the following equations, based on equations (3.1.5), (3.2.5.) and (3.3.5).

$$
\begin{gathered}
I_{1}=\frac{\frac{V_{1 a}}{r_{a}} \frac{r_{b}}{V_{1 b}}\left(1-e^{-\frac{\pi r_{a}}{\omega L} D}\right) e^{-\frac{\pi_{b}}{\omega L}(1-D)}+1-e^{-\frac{\pi_{b}}{\omega L}(1-D)}}{\frac{r_{b}}{V_{1 b}^{\prime}}+\frac{r_{b}}{V_{1 b}} e^{-\frac{\pi r_{a}}{\omega L} D} e^{-\frac{\pi r_{b}}{\omega L}(1-D)}} \\
I_{2}=\frac{\frac{V_{1 a}}{r_{a}} \frac{r_{b}}{V_{1 b}^{\prime}}\left(1-e^{-\frac{\pi_{a}}{\omega L} D}\right)+\left\{1-e^{-\frac{\pi r_{b}}{\omega L}(1-D)}\right\} e^{-\frac{\pi r_{b}}{\omega L} D}}{\frac{r_{b}}{V_{1 b}^{\prime}}+\frac{r_{b}}{V_{1 b}} e^{-\frac{\pi_{a}}{\omega L} D} e^{-\frac{\pi r_{b}}{\omega L}(1-D)}}
\end{gathered}
$$

3.4.2 Calculation for conduction loss of semiconductor device

Individual switching elements are in on-state only in half cycle. Further, the conduction loss caused in the positive half cycle is identical to that caused in the negative half cycle. Using $i 1$ and $i 2$ obtained in sections 3.1 through 3.3, as well as device model as shown in Fig. 5, individual conduction losses of IGBT PT1 and diode PD1 of INV1, as well as that of IGBT $P$ T2 and diode $P$ D2 of INV2, are given by the following equations:

$$
\begin{aligned}
P_{D 1} & =\frac{1}{2 T}\left[\int_{0}^{T_{1}}\left\{-i_{1 a}(t) \cdot v_{D}+i_{1 a}^{2}(t) \cdot r_{D}\right\} d t+\int_{0}^{T_{2}}\left\{-i_{1 b}(t) \cdot v_{D}+i_{1 b}^{2}(t) \cdot r_{D}\right\} d t\right] \\
& =\frac{1}{2}\left\langle\left(r_{D} \frac{V_{1 a}}{r_{a}}-v_{D}\right) \frac{V_{1 a}}{r_{a}} D\right. \\
& +\frac{\omega L}{\pi r_{a}}\left\{r_{D} \frac{I_{1}^{2}-I_{2}^{2}}{2}-\left(r_{D} \frac{V_{1 a}}{r_{a}}-v_{D}\right)\left(I_{1}+I_{2}\right)\right\} \\
& +\frac{\omega L}{\pi r_{b}}\left[\frac{r_{D} I_{2}^{2}}{2}+\left(r_{D} \frac{V_{1 b}}{r_{b}}-v_{D}\right)\left\{\left\{\frac{V_{1 b}}{r_{b}} \ln \left(1-\frac{r_{b} I_{2}}{V_{1 b}}\right)+I_{2}\right\}\right]\right\rangle
\end{aligned}
$$

$$
\begin{aligned}
P_{T 1} & =\frac{1}{2 T} \int_{0}^{T-T_{1}-T_{2}}\left\{i_{1 b}^{\prime}(t) \cdot v_{T}+i_{1 b}^{\prime 2}(t) \cdot r_{T}\right\} d t \\
& =\frac{1}{2} \frac{\omega L}{\pi r_{b}}\left[-\frac{r_{T} I_{1}^{2}}{2}-\left(r_{T} \frac{V_{1 b}^{\prime}}{r_{b}}+v_{T}\right)\left\{\frac{V_{1 b}^{\prime}}{r_{b}} \ln \left(1-\frac{r_{b} I_{2}}{V_{1 b}}\right)+I_{1}-\frac{\pi r_{b}}{\omega L} \frac{V_{1 b}^{\prime}}{r_{b}}(1-D)\right\}\right]
\end{aligned}
$$

$$
\begin{aligned}
P_{D 2}= & \frac{1}{2 T}\left[\int_{0}^{T_{1}}\left\{-i_{2 a}(t) \cdot v_{D}+i_{2 a}^{2}(t) \cdot r_{D}\right\} d t+\int_{0}^{T-T_{1}-T_{2}}\left\{i_{2 b}^{\prime}(t) \cdot v_{D}+i_{2 b}^{\prime 2}(t) \cdot r_{D}\right\} d t\right] \\
= & \frac{1}{2}\left\langle\left(r_{D} \frac{V_{1 a}}{r_{a}}-v_{D}\right) \frac{V_{1 a}}{r_{a}} D\right. \\
& +\frac{\omega L}{\pi r_{a}}\left\{r_{D} \frac{I_{1}^{2}-I_{2}^{2}}{2}-\left(r_{D} \frac{V_{1 a}}{r_{a}}-v_{D}\right)\left(I_{1}+I_{2}\right)\right\} \\
& \left.+\frac{\omega L}{\pi r_{b}}\left[-\frac{r_{D} I_{1}^{2}}{2}-\left(r_{D} \frac{V_{1 b}^{\prime}}{r_{b}}+v_{D}\right)\left\{\frac{V_{1 b}^{\prime}}{r_{b}} \ln \left(1-\frac{r_{b} I_{2}}{V_{1 b}}\right)+I_{1}\right\}\right]-\frac{\pi r_{b}}{\omega L} \frac{V_{1 b}^{\prime}}{r_{b}}(1-D)\right\rangle
\end{aligned}
$$

$$
\begin{aligned}
P_{T 2} & =\frac{1}{2 T} \int_{0}^{T_{2}}\left\{i_{2 b}(t) \cdot v_{T}+i_{2 b}^{2}(t) \cdot r_{T}\right\} d t \\
& =\frac{1}{2} \frac{\omega L}{\pi r_{b}}\left[\frac{r_{T} I_{2}^{2}}{2}+\left(r_{T} \frac{V_{1 b}}{r_{b}}-v_{T}\right)\left\{\frac{V_{1 b}}{r_{b}} \ln \left(1-\frac{r_{b} I_{2}}{V_{1 b}}\right)+I_{2}\right\}\right]
\end{aligned}
$$

\subsubsection{Calculation of DC power}

In power running mode, the power $P 1$ is transmitted from the DC power source $V 1$ and the power $P o$ is transmitted to the DC power source $V$ o. By making use of the rectification of the inverter, only an AC half cycle should be used. In state $a$, current transmitted from DC power source $V 1$ is zero. In state $b, i 1 \mathrm{~b}+i 2 \mathrm{~b}=2 i 1 \mathrm{~b}$, and in state $c, i^{\prime} 1 \mathrm{~b}+i^{\prime} 2 \mathrm{~b}=2 i^{\prime} 1 \mathrm{~b}$. The current which is transmitted to DC power source $V 0$ is $-i 2 \mathrm{a}$ in state $a, i 2 \mathrm{~b}$ in state $b$ and $i^{\prime} 1 \mathrm{~b}$ in state $c$, respectively. Therefore, power $P 1$ and power $P$ o are given by the following equations. 


$$
\begin{aligned}
P_{1}= & \frac{1}{T}\left\{\int_{0}^{T_{2}} 2 i_{1 b}(t) \cdot V_{1} d t+\int_{0}^{T-T_{1}-T_{2}} 2 i_{1 b}^{\prime}(t) \cdot V_{1} d t\right\} \\
= & 2 V_{1}\left[\frac{\omega L}{\pi r_{b}}\left\{\left(\frac{V_{1 b}}{r_{b}}-\frac{V_{1 b}^{\prime}}{r_{b}}\right) \ln \left(1-\frac{r_{b} I_{2}}{V_{1 b}}\right)-I_{1}+I_{2}\right\}+\frac{V_{1 b}^{\prime}}{r_{b}}(1-D)\right] \\
P_{O} & =\frac{1}{T}\left\{\int_{0}^{T_{1}}-i_{2 a}(t) \cdot V_{O} d t+\int_{0}^{T_{2}} i_{2 b}(t) \cdot V_{O} d t+\int_{0}^{T-T_{1}-T_{2}} i_{2 b}^{\prime}(t) \cdot V_{O} d t\right\} \\
& =V_{O}\left[-\frac{V_{1 a}}{r_{a}} D+\frac{V_{1 b}^{\prime}}{r_{b}}(1-D)+\frac{\omega L}{\pi r_{a}}\left(I_{1}+I_{2}\right)\right. \\
& \left.+\frac{\omega L}{\pi r_{b}}\left\{\left(\frac{V_{1 b}}{r_{b}}-\frac{V_{1 b}^{\prime}}{r_{b}}\right) \ln \left(1-\frac{r_{b} I_{2}}{V_{1 b}}\right)-I_{1}+I_{2}\right\}\right]
\end{aligned}
$$

\subsubsection{Power efficiency}

The power efficiency $\mathrm{y}$ of the proposed DC-DC converter in power running mode is defined by operation (3.4.9). The iron loss of the transformer can be calculated by considering the core loss characteristics provided by a core manufacturer under the conditions: magnetic flux density of $0.267 \mathrm{~T}$ (= $V_{\mathrm{o} T / 4 N A} ; \mathrm{N}=$ number of turns; $\mathrm{A}=$ iron core cross-section area $\mathrm{m}^{2}$ ), operating frequency of $10 \mathrm{kHz}$, and operating temperature of $60^{\circ} \mathrm{C}$. Based on the characteristics of devices used in the circuit, the switching losses corresponding to individual current during the turn-on and turn-off states are calculated, respectively. In power regenerating mode, power $P o$ is replaced by power $P 1$.

$$
\eta=\frac{P_{O}}{\left(P_{1}+\text { iron loss of transformer }+ \text { switching loss of swiching devices }\right)}
$$

\subsubsection{Effective current of transformer coil}

The effective current of the transformer coil is given by means of individually squaring the value of $i 1$ in state $a, b$ and $c$, integrating them to obtain the total value, and then dividing the total value by time $T$. Its square root is defined as the effective value I1 rms of the primary coil current. The turn ratio of the transformer is unity, that is, the effective value I $2 \mathrm{rms}$ of the secondary coil current is equal to I $1 \mathrm{rms}$.

$$
\begin{aligned}
& I_{1 r m s}=\sqrt{\frac{1}{T}\left\{\int_{0}^{T_{1}} i_{1 a}^{2}(t) d t+\int_{0}^{T_{2}} i_{1 b}^{2}(t) d t+\int_{0}^{T-T_{1}-T_{2}} i_{1 b}^{\prime 2}(t) d t\right\}=} \\
& \sqrt{\left(\frac{V_{1 a}}{r_{a}}\right)^{2} D+\left(\frac{V_{1 b}^{\prime}}{r_{a}}\right)^{2}(1-D)+\frac{\omega L}{\pi r_{a}}\left\{\frac{I_{1}^{2}-I_{2}^{2}}{2}-\frac{V_{1 a}}{r_{a}}\left(I_{1}+I_{2}\right)\right\}} \\
& \sqrt{+\frac{\omega L}{\pi r_{a}}\left[\left\{\left(\frac{V_{1 a}}{r_{a}}\right)^{2}-\left(\frac{V_{1 b}^{\prime}}{r_{a}}\right)^{2}\right\} \ln \left(1-\frac{r_{b} I_{2}}{V_{1 b}}\right)+\frac{I_{1}^{2}-I_{2}^{2}}{2}+\frac{V_{1 b}}{r_{b}} I_{2}-\frac{V_{1 b}^{\prime}}{r_{a}} I_{1}\right]}
\end{aligned}
$$

\section{Analytical and Experimental results}

A similar analysis is performed in other operating modes just in power regenerating and running modes. Further, phaseshift duty ratio $D(=T 1 / T)$ is defined as a parameter, and then

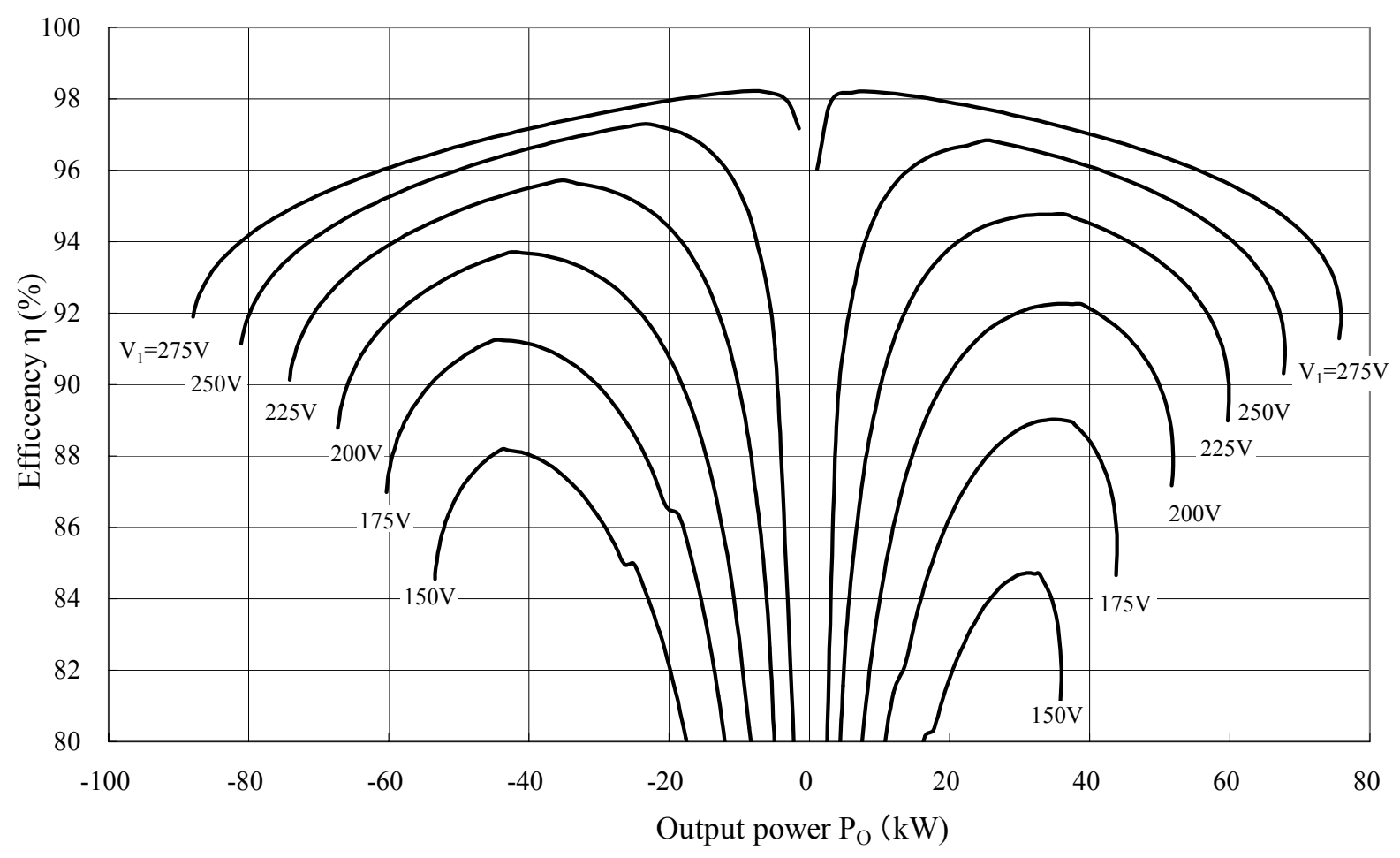

Fig. 9 Output $\mathrm{P}_{\mathrm{o}}$ vs. efficiency $\eta$ 


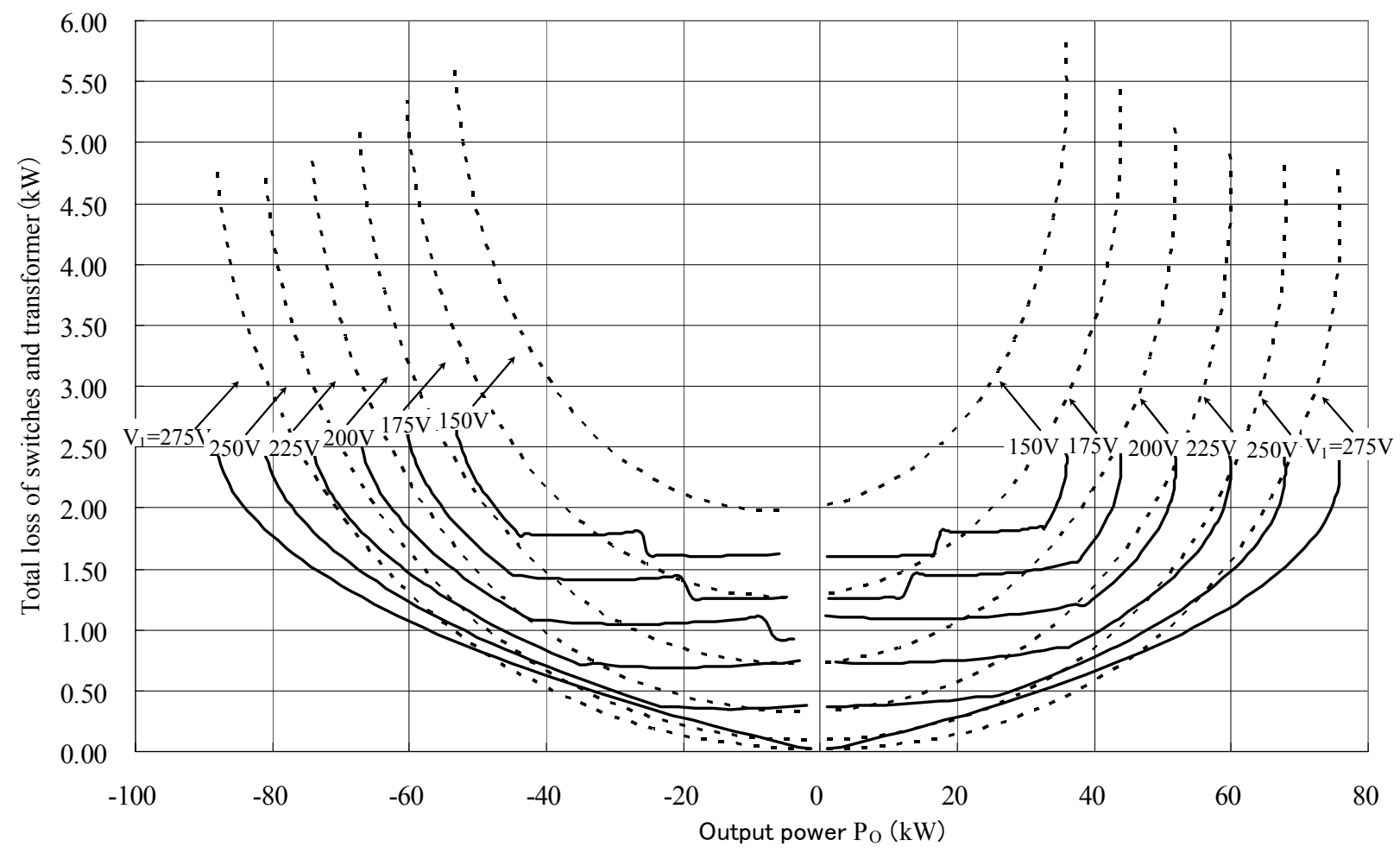

Fig. 10 Output $\mathrm{P}_{\mathrm{o}}$ vs. total device loss and transformer

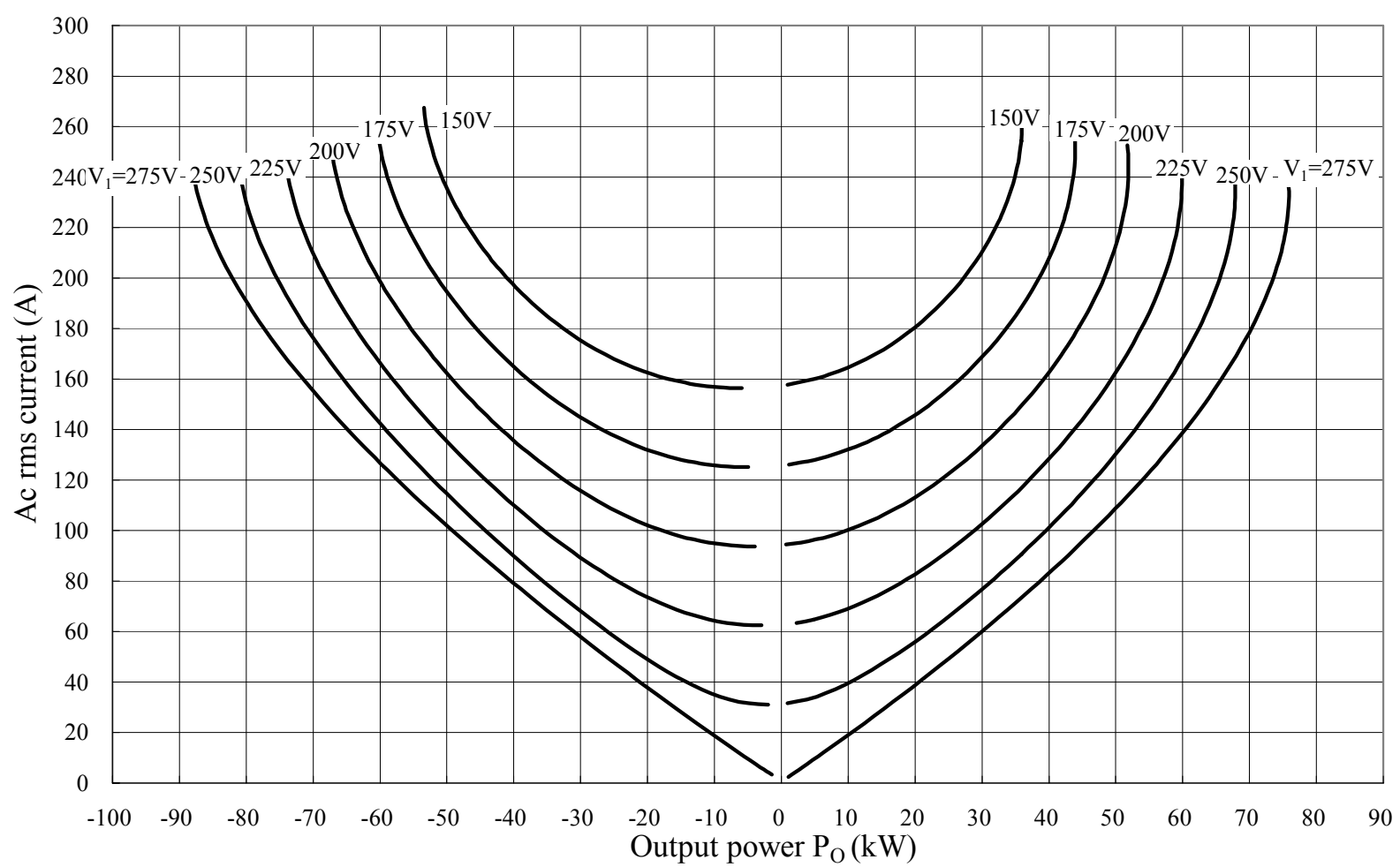

Fig. 11 Output $\mathrm{P}_{\mathrm{o}}$ vs. ac rms current 


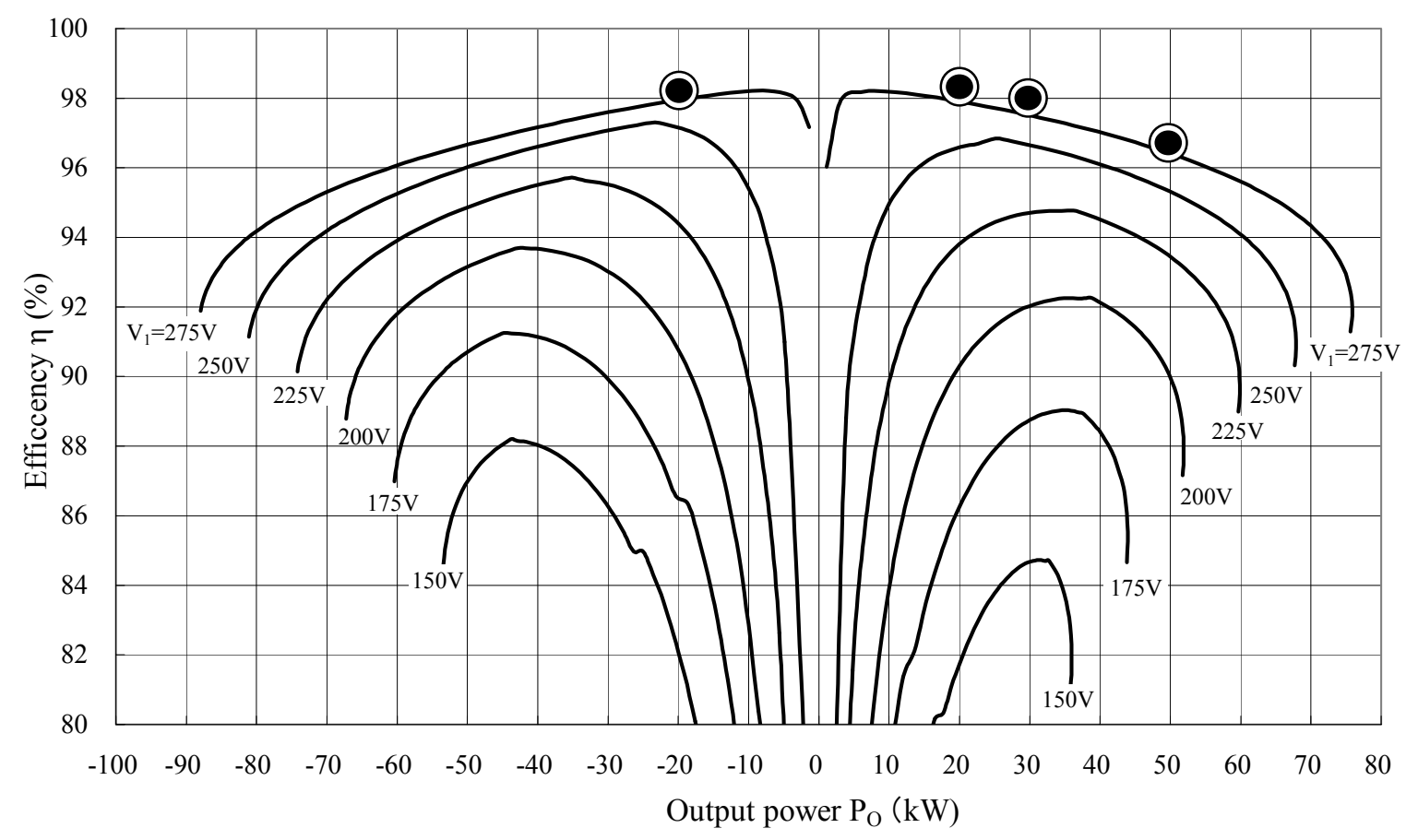

Fig. 12 Output $\mathrm{P}_{\mathrm{o}}$ vs. efficiency $\eta$

$I 1$ and $I 2$ are given by the equations equivalent to the equations (3.4.1) and (3.4.2). The values of $I 1$ and $I 2$ are assigned to equations equivalent to equations (3.4.3) through (3.4.7) in individual states, to calculate power, loss, efficiency and $\mathrm{AC}$ effective value. Fig. 9 shows the $P$ o vs. efficiency characteristic, by means of placing the output power (Power $P$ o of $\left.V_{\mathrm{O}}\right)$ as a transverse axis. Fig. 10 shows the $P$ o vs. total loss of semiconductor devices and transformer loss characteristic. Fig. 11 shows the $P$ o vs. AC effective value. The switching loss is included in the semiconductor device loss.

The regions increased in a staircase pattern appear in Fig. 10. This phenomenon occurs because superconductor devices used in the circuit (PM300CLA060 manufactured by Mitsubishi Electric) act as a 2-stage switching for turn-on time by making the border at a collector current of 150A. In the regions of lower current, the hard-turn-on phenomenon of the switching device is often occurs, in this case, the power loss is small because power loss is minor. Then, the conduction loss is also small.

As shown in Fig. 9, the lower DC power source voltage $V 1$ in power regenerating and running modes is, the lower power efficiency is, resulting in a steep decrease in processable maximum power. In this circuit, a constant rating of approx. $20 \mathrm{~kW}$ can be processed in power running mode, while, processing up to $90 \mathrm{~kW}$ is made possible for a short time in power regenerating mode. In case of a constant rating of $20 \mathrm{~kW}$ and $V 1=275 \mathrm{~V}$, power efficiency of $98 \%$ or higher can be obtained. In the case of a constant rating of $75 \mathrm{~kW}$ and up to $V 1=225 \mathrm{~V}, 90 \%$ or higher can be obtained. A maximum efficiency of $89.9 \%$ can be obtained in the experiment using a second-quadrant chopper as shown in Fig. 1, under the same conditions.
It is seen in Fig. 10 that the lower DC power source voltage $V 1$ is and/or the higher electric power is, the larger steeply the total loss of the transformer is. Although the loss of the transformer at $V 1=275 \mathrm{~V}$ in a constant rating is approx. $1 \%$, the design of the transformer should be designed carefully in the case of long time use under the wide range conditions of power and voltage.

The experimental output power vs. power efficiency characteristics is shown in Fig.12. The symbol $\bullet$ shows the observed results. It is seen in Fig. 9 and Fig. 12 that theoretical power efficiency is agreed well with the experimental one, and that the very high power efficiency can be obtained.

\section{Conclusions}

An AC-link bi-directional DC-DC converter is proposed. Even if the DC voltage $V_{\mathrm{O}}$ is $550 \mathrm{~V}, 600 \mathrm{~V}$ rated switching devices can be used. By decreasing current flowing to switching devices and transformer by half, the power efficiency of $98 \%$ or higher at a constant rating can be obtained. The down-sizing of the inductor for circuit operation can be actualized by only making use of the leakage inductance of the transformer. The very effective performance is obtained by the proposed bidirectional DC-DC converter in practical use.

\section{REFERENCES}

[1] Nikkei Monozukuri: "Motor Control Techniques Being Compatible with Eco and Power in TOYOTA," pp. 68-73, Nikkei BP Co. Ltd.(August 2004)

[2] Institute of Electrical Engineering, Investigation Committee of the Semiconductor Power Conversion: "Semiconductor Power Convention Circuit," p. 80, Ohm Co. Ltd.

[3] Shigemori Inoue and Hirofumi Akagi: "Bidirectional Isolation Type DC/DC Converter," Institute of Electrical Engineering Technical Report, EOD-04-59/SPC-04-121,pp.54-64(April 2004) 